\title{
SEVERE HYPOGLYCEMIA DURING PREGNANCY: ITS FREQUENCY AND PREDISPOSING FACTORS IN DIABETIC WOMEN
}

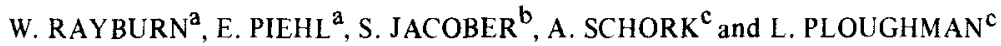 \\ a Division of Maternal Fetal Medicine, Department of Obstetrics and Gynecology, ${ }^{b}$ Division of Endocrinology. Department \\ of Internal Medicine, University of Michigan Medical School and ${ }^{\mathrm{C}}$ Department of Biostatistics, School of Public Health, University \\ of Michigan, MI (USA)
}

(Received October 28th, 1985)

(Accepted March 26th, 1986)

\section{Abstract}

Rayburn W, Piehl E, Jacober S, Schork A, Ploughmann L (Division of Maternal Fetal Medicine, Department of Obstetrics and Gynecology, Division of Endocrinology. Department of Internal Medicine, University of Michigan Medical School and Department of Biostatistics, School of Public Health, University of Michigan, MI, USA). Severe hypoglycemia during pregnancy: Its frequency and predisposing factors in diabetic women.

Int J Gynaecol Obstet 24: 263-268, 1986

Severe hypoglycemic episodes, as defined as altered consciousness to the extent that self treatment is impossible, were sought prospectively in pregnant diabetic women. One or more episodes were found in none of 21 gestational onset, insulin-requiring women during their 28 pregnancies but were present in 19 (33\%) of the 57 already insulin dependent (Type 1) women during $26(36 \%)$ of their 72 pregnancies. The most common predisposing factors included strict glucose control, anorexia, early morning hours (1200-0900), lack of an adrenergic response and time shortly before the next anticipated meal.

Keywords: Hypoglycemia; Diabetes; Pregnancy; Insulin.

\section{Introduction}

As more diabetic women become pregnant, a greater emphasis is being placed on optimal perinatal outcomes. The trend towards improved fetal survival is largely attributed to stricter metabolic control before and during gestation, since most complications are conditioned by maternal glucose levels [1]. Furthermore, a considerable body of clinical and scientific data has supported the contention that meticulous management may delay or prevent complications in the diabetic woman [9].

Avoidance of hyperglycemia, rather than hypoglycemia, is generally stressed as the first priority during pregnancy. In striving for strict metabolic control, it is expected that pregnant diabetic women are at increased risk for hypoglycemia. Blood glucose levels at these times are lower than the lowest limit of normal physiologic fluctuations, which in many adults is between $40-60 \mathrm{mg} \%$ [5]. The release of glucagon and catecholamines (primarily epinephrine) act as overlapping glucoregulatory systems to prevent or correct hypoglycemia [10].

Once low glucose levels are reached, maternal symptoms or signs become apparent either from an adrenergic defense response or from cellular malnutrition at the neuronal level [5]. Adrenergic symptoms predominate in reproductive age persons without long 
standing diabetes $[5,10]$. These symptoms include shakiness, palpitations, tachycardia, irritability, sweating, hunger and perioral paresthesias. Therapy to reverse these symptoms includes the self-administration of orange juice, commercial glucose concentrates, honey, sugar, candy, cola drinks and milk.

When this warning is absent, symptoms from neuronal cellular malnutrition may develop. Short-term central nervous system dysfunction may lead to headache, loss of concentration, confusion, visual disturbance, stupor and unconsciousness. Convulsions, coma and even death may supervene without prompt treatment [5].

A search of the obstetric literature has not disclosed descriptions of severe maternal hypoglycemia. The purpose of this study was to determine the frequency of severe hypoglycemia during pregnancy and to better understand the predisposing conditions. In doing so, preventative medical care or prompt medical treatment may be offered.

\section{Materials and methods}

Women eligible for this investigation included those who were already insulindependent (Type 1) before conception and those who required insulin for the first time during gestation. Each person attended a weekly high risk obstetric clinic during the $3 \frac{1}{2}$ year period between July, 1981 and December, 1984. Pregnancies aborting before the 12 th week were excluded. Care of these women was overseen by both obstetric and endocrine staff members. Attempts were made to attain strict glucose control as determined from daily capillary blood glucose determinations and standardized for our clinic [6]. Control was classified as strict if preprandial glucose values averaged to be 100 $\mathrm{mg} / \mathrm{dl}$ or less, fair if $101-149 \mathrm{mg} / \mathrm{dl}$, and poor if $150 \mathrm{mg} / \mathrm{dl}$ or more [6].

Data about hypoglycemic episodes were gathered prospectively. Monitoring of blood glucoses by the patient was undertaken using reflectance photometers or visually-read blood glucose oxidase strips. A standard form was used by the mothers to record the insulin dose and capillary blood glucose values obtained four times daily. Each person was asked to record the time of day and events surrounding any symptomatic hypoglycemic episodes in the space provided on the data sheet. Therapy to relieve these symptoms was also recorded. Additional findings were ascertained with each weekly or bi-weekly clinic visit and recorded on the antepartum chart.

Hypoglycemic reactions were classified as mild or severe. Mild reactions were defined as hypoglycemic episodes that could be self treated. These episodes consisted of adrenergic signs and symptoms as described previously. Severe reactions were defined as hypoglycemia leading to such an altered consciousness (loss of concentration, confusion, stupor, unconsciousness) that another person was required to administer glucose or glucagon [7]. These severe hypoglycemic episodes were often preceded by a lack of warning adrenergic symptoms. Conditions known to predispose to severe hypoglycemia in the non-pregnant person were sought by the investigators and recalled easily by the patient, close friend or family member $[2,3,5,7,9,10]$.

Comparisons between women categorized as having severe hypoglycemia or not were made using the chi-square and Student's $t$-tests and the analysis of variance. A $P$-value less than 0.05 was considered to be statistically significant.

\section{Results}

Seventy-eight diabetic women sought our care for 100 pregnancies during the $3 \frac{1}{2} 2$-year period. Fifty-seven $(73 \%)$ of these patients with 72 pregnancies were already insulin dependent, having received therapy before conception for $11 \pm 5$ years (mean \pm 1 S.D.). These insulin-dependent women were followed for $24 \pm 4$ weeks gestation and delivered 
at $37 \pm 1$ weeks. The other $21(27 \%)$ women with 28 pregnancies had gestational onset diabetes and required insulin for $14 \pm 3$ weeks. They were followed for $22 \pm 3$ weeks and delivered at $39 \pm 1$ weeks. Severe hypoglycemic episodes did not occur during pregnancy for any of these gestational-onset diabetics.

Compared with before conception, pregnancy was found to predispose to severe hypoglycemia in insulin-dependent diabetics. Thirty-five severe hypoglycemic episodes were reported. These occurred in $19(33 \%)$ of the 57 already insulin-dependent women during $26(36 \%)$ of the 72 pregnancies. The number of severe hypoglycemic reactions per patient month is shown in Fig. 1. Severe hypoglycemia occurred with much less frequency (0.08 episode/month) than symptomatic mild hypoglycemia (5.0 episodes/month). A recurrence of severe hypoglycemia during pregnancy was found in eight of these 19 women. Conditions associated with these recurrences included the desire for strict metabolic control, a lack of a prior adrenergic response, and a missed meal.

Conditions predisposing to severe hypoglycemia were similar to those associated with mlde hypoglycemia. These factors

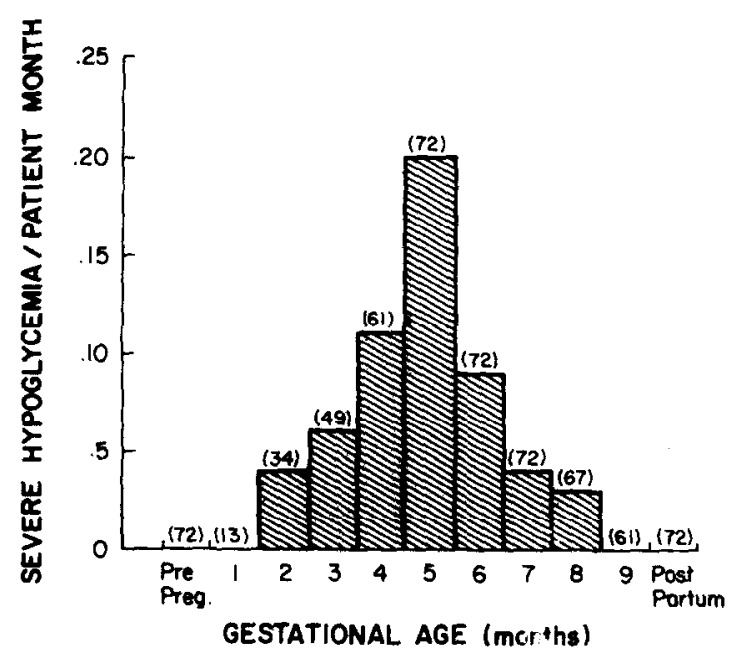

Fig. 1. Severe hypoglycemic episodes per patient morith for insulin-dependent diabetic women during pregnancy. (Number in parentheses represents number of pregnancies).
Table 1. Conditions predisposing to severe hypoglycemia on 35 occasions during pregnancy. (These conditions w'ere often not found to be inutually exclusive of each other).

\begin{tabular}{lll}
\hline Condition & No. & $(\%)$ \\
\hline Strict glucose control & 21 & $(60)$ \\
Anorexia & 15 & $(43)$ \\
Early morning hours (1200-0800) & 14 & $(40)$ \\
Lack of a prior adrenergic response & 14 & $(40)$ \\
Two hours before an anticipated meal & 13 & $(38)$ \\
Poor glucose control & 8 & $(23)$ \\
Missed meal & 6 & $(17)$ \\
Excess activity & 5 & $(14)$ \\
Insulin overdose & 5 & $(14)$ \\
Recent severe hypoglycemia & 4 & $(14)$ \\
Recent hospitalization & 4 & $(14)$ \\
\hline
\end{tabular}

included strict glucose control, anorexia, early morning hours (1200-0800), lack of an adrenergic response, and $2 \mathrm{~h}$ before the next anticipated meal (Table I). All of these factors were not mutually exclusive of each other. Pregnancy profiles of the already insulin-dependent women are compared between those in which severe hypoglycemia was present or absent (Table II). The maternal age, race, parity, prepregnancy insulin dose,

Table II. Profiles of insulin-dependent diabetic women according to the presence or absence of severe hypoglycemia during pregnancy.

\begin{tabular}{lcc}
\hline & \multicolumn{2}{c}{ Severe hypoglycemia } \\
\cline { 2 - 3 } & $\begin{array}{l}\text { Present } \\
(N=26)\end{array}$ & $\begin{array}{l}\text { A bsent } \\
(N=46)\end{array}$ \\
\hline Maternal age (years) & $24 \pm 5$ & $26 \pm 4$ \\
Race (no. caucasian) & $24(92 \%)$ & $41(89 \%)$ \\
Parity (no. primiparous) & $15(58 \%)$ & $34(74 \%)$ \\
Duration of diabetes (years) & $12 \pm 5$ & $11 \pm 7$ \\
Prepregnancy insulin dose & $46 \pm 15$ & $41 \pm 17$ \\
$\quad$ (total units per day) & & \\
Gestational age & $11 \pm 4$ & $12 \pm 4$ \\
$\quad$ Initial prenatal visit weeks) & $36 \pm 12$ & $37 \pm 1$ \\
$\quad$ Delivery (weeks) & & \\
Glucose control before delivery & $\quad 11(42 \%)$ & $24(52 \%)$ \\
$\quad$ Strict & $10(39 \%)$ & $13(28 \%)$ \\
$\quad$ Pair & $5(19 \%)$ & $9(20 \%)$ \\
\hline
\end{tabular}

${ }^{\text {a }}$ Difference in all comparisons were not found to be statistically different $(P>0.05)$. 
duration of diabetes, duration of prenatal care, and extent of metabolic control late in gestation were not statistically different between the two groups.

Therapy for any unconscious mother consisted of either the intramuscular administration of $1 \mathrm{ml}$ of glucagon by a family member or the infusion of glucose using $25-50 \mathrm{ml}$ of a $50 \%$ glucose solution in an emergency room. A return to consciousness was apparent with prompt treatment. Adverse maternal effects were found in the two cases described below when therapy was begun after prolonged severe hypoglycemia.

\section{Case 1}

A 24-year-old $G_{2} P_{0}$ woman with class $R$ diabetes and a prior history of poor glucose control was evaluated initially at 11 weeks gestation. She continued to have early morning nausea following the initial hospitalization, in which her glucose control become fair (capillary glucose levels averaging between $101-150 \mathrm{mg} / \mathrm{dl}$. On the day of her emergency hospitalization at 12 weeks gestation, she had taken her usual morning insulin dose (36 units NPH, 8 units regular insulin) but did not eat breakfast or a late morning snack. Her fiance last spoke with her at noon. She was found unconscious with vomitus in her mouth at about 1700 . Her serum glucose level in the emergency room was $33 \mathrm{mg} / \mathrm{dl}$, and she became more conscious following the infusion of 25 of $50 \%$ dextrose in water. Her initial tests revealed an arterial $P_{2}$ of $35 \mathrm{mmHg}$ and diffuse bilateral pulmonary infiltrates on chest X-ray. Therapy was undertaken for apparent adult respiratory distress from aspiration of vomitus. She became increasingly difficult to oxygenate despite intubation, positive-end expiratory pressures with high concentrations of oxygen, antibiotics, and supportive measures. She aborted spontaneously, underwent repeated severe hypoxic episodes, and subsequently died within 11 days of admission.
Case 2

A 22-year-old $G_{3} \quad P_{0} \mathrm{SAb}_{2}$ woman sustained hypoglycemia induced grand mal seizures. Eight months before gestation her first and only seizurs occurred after developing a low blood glucose level. A neurologic evaluation was unremarkable. Her diabetes had been present for 13 years, and she was seen initially at 9 weeks gestation. The 47 units of insulin were administered daily by continuous subcutaneous infusion and intermittent subcutaneous injection. Her glucose control was excellent with a hemoglobin $\mathrm{A}_{1} \mathrm{C}$ of 6.6 and strict control (averaged glucose values below $100 \mathrm{mg} / \mathrm{dl}$ during the initial hospitalization. During the 12 th week, she sustained a grand mal seizure at 0900 following her usual insulin therapy and a small serving of cereal. A capillary glucose determination of $27 \mathrm{mg} \%$ was similar to that recorded earlier in the week without any apparent warning symptoms. The nausea and vomiting of pregnancy were the only other predisposing factors. An infusion of glucose at the nearby emergency room caused the tonic-clonic seizure activity to subside.

The perinatal outcomes of the insulindependent women sustaining severe hypoglycemia were similar to those without

Table III. Perinatal outcomes of insulin-dependent women according to the presence or absence of severe hypoglycemic episodes during pregnancy.

\begin{tabular}{lll}
\hline & \multicolumn{2}{l}{ Severe hypoglycemia } \\
\cline { 2 - 3 } & $\begin{array}{l}\text { Present } \\
(N=26)\end{array}$ & $\begin{array}{l}\text { Absent } \\
(N=46)\end{array}$ \\
\hline Gestational age & $15(58 \%)$ & $30(65 \%)$ \\
$\quad<37$ weeks & $2992 \pm 453$ & $3245 \pm 646$ \\
Birth weight $(g)$ & $8(31 \%)$ & $22(48 \%)$ \\
Large for gestational age & $7 \pm 2$ & $7 \pm 1$ \\
1-min Apgar score & $8 \pm 1$ & $8 \pm 1$ \\
5-min Apgar score & $1(4 \%)$ & $2(4 \%)$ \\
Major anomalies &
\end{tabular}

${ }^{a}$ Differences in all comparisons were not found to be statistically significant $(P>0.05)$. 
such episodes. The gestational age at delivery, route of delivery, birth weight, 1- and 5minute Apgar scores, and percent of anomalous infants were not significantly different (Table III). The only stillborn infant in all the diabetic women was delivered at 31 weeks gestation before fetal monitoring was undertaken. This 20-year-old primigravid mother with class $\mathrm{C}$ diabetes had poor glucose control in early gestation and sustained two severe hypoglycemic episodes at 13 and 15 weeks gestation. Later in pregnancy, her metabolic control was much improved with daily glucose monitoring. An autopsy of the normal-appearing infant was unremarkable, and histologic examination of the placenta revealed chronic vascular changes.

\section{Discussion}

A diabetic woman undergoing pregnancy must be educated in attempting to achieve a fine balance between strict glucose control and mild hypoglycemic episodes. Most of these persons had never attempted such close monitoring of their blood glucose levels. Pregnancy was found to be associated with more frequent episodes of severe hypoglycemia in women who were already insulindependent. A recent review of five centers by Santiago et al. [7] in non-pregnant diabetics revealed the frequencies of severe hypoglycemia to be $0.005-0.103 /$ patient month. This is similar to the $0.08 /$ patient month during pregnancy in the present study.

Because of improvements in screening for diabetes and treatment during pregnancy, more women are being treated with insulin and for longer periods during pregnancy. None of the gestational onset, insulin-requiring diabetic women in this study encountered any severe hypoglycemia. Symptoms from adrenergic responses were sufficient for hypoglycemia to be detected before it became severe. Once these persons became aware of these warning symptoms, relief with self-administration of oral glucose supple- ments was all that was necessary. Many of these gestational diabetics required large daily doses of insulin, and an insulin overdose remains a theoretic concern.

Defective recovery from insulin-induced hypoglycemia was found to occur in onethird of the already insulin-dependent women during pregnancy. This finding is higher than the $4 \%$ reported by Goldstein et al. [2] and the $26 \%$ by Goldwicht et al. [3] in children and young adults. Those investigators also found that bouts of frequent or severe symptomatic hypoglycemia may be more common when diabetes is well-controlled during intensive therapy. The previously insulindependent diabetic women in this study were generally very conscientious about their diabetes self-care and maintained fair or strict glucose control. Difficulties with eating or missing a meal would be explained by early gestation nausea, a lack of desire to increase caloric intake and viral gastroenteritis.

Perinatal outcomes for insulin-dependent women in this study were similar regardless of the presence or absence of severe hypoglycemia. This reassurance is limited by the small number of pregnancies and the evaluation of infants in the newborn period only. Animal studies by Kalter and Warkany have revealed no increased long-term structural defects in offspring following hypoglycemic shock during an insulin overdose [4]. This contrasts with a report by Sobel in 1960 in which two children were reported to have multiple defects following the treatment of 17 mothers with insulin shock for psychiatric illness [8].

The authors conclude that pregnancy in already insulin-dependent diabetic women predisposes to a greater risk of severe hypoglycemia. It is difficult to predict this before gestation, but conditions in which there is inadequate caloric intake or a lack of an alarming adrenergic response should be considered. Prompt reversal of any altered central nervous function with glucose or glucagon therapy and subsequent modi- 
fication of therapeutic goals (permitting mild fasting hyperglycemia, more frequent glucose monitoring, patient re-education, insulin infusion screening tests) are necessary to avoid future catastrophic consequences.

\section{References}

1 Gabbe $\dot{S}$ : Diabetes mellitus in pregnancy. In Fetal and Maternal Medicine (E Quilligan, N Kretchmer eds), pp 587-608. Wiley Medical Publication, 1980.

2 Goldstein D, England V, Hess $\mathbf{R}$ et al.: A prospective study of symptomatic hypoglycemia in young diabetic patients. Diab Care 4: 601, 1981.

3 Goldwicht C, Stama G, Papoe L, et al.: Hypoglycemia reactions in 172 type I (insulin-dependent) diabetic patients. Diabetologia 24: 95, 1985.

4 Kalter H, Warkany J: Experimentation production of congenital malformations in mammals by metabolic procedure. Physiol Rev 39: 69, 1959.

5 Lefebyre PJ and Luyckx AS: Hypoglycemia. In Diabetes Mellitus, Theory and Practice. (M Ellenberg, HR Rifkin eds), 3rd edn, pp 987-1004. Medical Examination Publishing Co., New York, 1983.

6 Rayburn W, Piehl E, Lewis E et al.: Changes in insulin therapy during pregnancy. Am J Perinatol 2: 271, 1985.

7 Santiago V, White N, Skor $D$ et al.: Defective glucose counter-regulation limits intensive therapy of diabetes mellitus. Am J Physiol 246: E 215, 1984.

8 Sobel DE: Fetal damage due to ECT, insulin coma, chlorpromazine or reserpine. AMA Arch Gen Psychol 2: $606,1960$.

9 Unger RH: Meticulous control of diabetes: Benefits, risks, and precautions. Diabetes 31: 479, 1982.

10 Unger RH: Insulin-glucagon relationships in the defense against hypogly cemia. Diabetes 32: 575, 1983.

Address for reprints:

William Raybum, M.D.

Department of Obstetrics \& Gynecology

University of Nebraska Medical Center

42nd \& Dewey Avenue

Omaha, NE 68105, USA 